\title{
Biological evaluation of PRGD/PDLLA for nerve regeneration scaffold
}

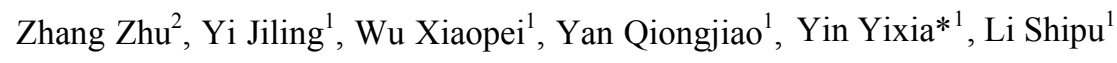 \\ (1: Biomedical Materials and Engineering \\ Research Center, Wuhan University of \\ Technology, Wuhan 430070, P.R.China; \\ 2: Affiliated hospital of Wuhan University of \\ Technology, Wuhan 430070, P.R.China)
}

\begin{abstract}
Objective: To develop a novel scaffold for nerve regeneration and explore its biocompatibility through culturing Schwann cells (SCs) on it an in vivo implantation. Methods: A novel scaffold of PDLLA with arginine-glycine-aspartic acid (RGD) peptide (PRGD/PDLLA) was synthesized.The proliferation of Schwann cells (SCs) concultured with PDLLA films (control group) and PRGD/PDLLA films were evaluated by MTT assay and scanning electron microscope observation. Then, histological assessment and ELISA testing on inflammatory-related cytokines TGF- $\beta 1$ were performed. Results: compared with PDLLA, PRGD/PDLLA films possesses better hydrophilicity, biocompatibility, degradation property and less inflammatory reaction. Conclusion: The present study indicated that PRGD/PDLLA scaffolds would meet the requirements of artificial nerve scaffold and have a potential application in the fields of nerve regeneration.
\end{abstract}

Key words: Schwann cells; PRGD/PDLLA; Implantation; Inflammatory factors; Biocompatibility

\section{INTRODUCTION}

Poly-d,1-lactic acid (PDLLA) is widely investigated for nerve regeneration $[1,2]$ due to its excellent biocompatibility, biodegradable and suitable mechanical properties. However, PDLLA is hydrophobic and lacks cell biological recognition and a friendly interface with cells. Arginine-glycine-aspartic acid (RGD) is found crucial in cell-binding domains of extracellular matrix proteins [3]. This peptide is also important for scaffolds to bionic the ingredients of extracellular matrix. Suitable materials for tissue engineering should possess good biocompatibility and degradation property and lower or no inflammatory-reaction. Cell adhesion ability of PDLLA is undesirable. Therefore, PRGD/PDLLA was synthesized, Schwann cells (SCs) were co-cultured with PRGD/PDLLA films and PDLLA films. The phenotypes of SCs such as cell proliferation, morphology in both PDLLA and PRGD/PDLLA films culture were observed and characterized. Animal implantation and histological assessment was evaluated, with a purpose to illustrate the potentiality of PRGD/PDLLA as artificial nerve scaffold.

\footnotetext{
* corresponding author : State Key Laboratory of Advanced Technology for Materials Synthesis and Processing, Wuhan University of Technology, Wuhan 430070, P.R. China

E-mail address: yinyx71@126.com
}

\section{MATERIALS AND METHODOLOGY}

\section{A. Preparation of PDLLA, PRGD/PDLLA films}

PRGD/PDLLA synthesis: The polymer RGD peptide modification of poly \{(lactic acid)-co-[(glycolic acid)alt-(L-lysine)]\} (PRGD) was synthesized by the following steps. Firstly, (3S)-3-[4(benzyloxycarbonylamino) butyl] morpholine-2, 5-dione (BMD) was synthesized by bromoacetyl bromide and Ne-(benzyloxycarbonyl)-L-lysine. Secondly, poly \{lactic acid)-co-[(glycolic acid)-alt- (NEbenzyloxycarbonyl -L-lysine)]\} (PLGLZ) was obtained by copolymerization of $\mathrm{D}$, L-lactide and BMD. Then, poly $\{$ (lactic acid)-co-[(glycolic acid)-alt-(L-lysine)]\} (PLGL) was synthesized by catalytic hydrogenation of PLGL. Finally, PLGL was modified with RGD peptide [4]. PDLLA, PRGD/PDLLA films were prepared by casting the corresponding solution in dichloromethane in $150 \mathrm{~mm}$ glass dishes. The solvent was evaporated at ambient temperature and finally dried under a vacuum for $48 \mathrm{~h}$.

\section{B. Contact angle measurement.}

The change of the hydrophilicity of PDLLA films and PRGD/PDLLA films was characterized by measuring the contact angles in air using a JC2000A (Shanghai Zhongchen, China) contact angle meter. A drop of water $(5 \mu \mathrm{L})$ was introduced on the films, and the contact angle was measured immediately the image of the water drops.

\section{Proliferation of SCs on the surface of films}

Based on previous studies[5, 6], SCs were isolated from peripheral nerve of neonatal Wister rat. Based on the different attachment rates of SCs and fibroblasts, SCs were purified. Cell viability on the films surface was characterized by MTT assay. After culturing for 1 day, 3 day, 5 day, 7 day respectively, the upper liquid was removed. $20 \mu \mathrm{L}$ MTT solution $(5 \mathrm{mg} \mathrm{mL}-1)$ was added to each culture well and incubated at $37{ }^{\circ} \mathrm{C}$ under $5 \% \mathrm{CO} 2$ conditions for $4 \mathrm{~h}$. The upper liquid was removed. DMSO was added to each culture well. The absorbance at $560 \mathrm{~nm}$ was recorded by an automatic enzyme scanner (Thermo Lab systems, Finland). Five parallels for every sample were averaged.

\section{Scanning electron microscopy of cultured SCs}

Scanning electron microscope (SEM) was used to visualize their surface feature of co-cultured films. SCs were fixed for $1 \mathrm{~h}$ in $2.5 \%$ glutaraldehyde and $0.5 \%$ paraformaldehyde in $0.1 \mathrm{M}$ phosphate buffer $(\mathrm{pH} 7.2)$. Following washing with buffer, cultures were post-fixed for $1 \mathrm{~h}$ with $1 \%$ osmium tetraoxide. Following another buffer wash, they were dehydrated in an ascending 
series of ethanol washes, at which point they were critical point dried by Freon. Surface coating of

\section{E. Animal implantation and histological assessment}

All procedures were performed in the Experimental Animals Breeding and Research Center of Hubei province, P.R. China. Animal care was conducted with the prior approval of the Animal Experimental Ethics Committee of Hubei province, P.R.China.

Twenty male Wister rats (Tongji Medicinal School, Huazhong University of Technology. ) , weighting between 250 and $280 \mathrm{~g}$ were randomly assigned to 2 equal groups (Totally $n=10$ ). Anesthetize the rats with intraperitoneal injection of $30 \mathrm{mg} / \mathrm{kg}$ of xylazin hydrochloride. Following hair shaving and skin disinfection with Betadine solution, skin was exposed through muscle splitting incision. All operations were performed by the same surgeon.

Samples were fixed with $4 \%$ paraformaldehyde in $10 \%$ sucrose solution for $2 \mathrm{~h}$. After immersion in $30 \%$ sucrose solution at $4^{\circ} \mathrm{C}$ for $24-48 \mathrm{~h}$, tissues were embedded in optimal cutting temperature compound and cut into 5 - $\mu$ $\mathrm{m}$ sections for hematoxylin andeosin (HE) staining.

\section{F. ELISA testing on cytokines on serum}

Blood of mice was harvested after scaffold implantation for 1 , 5week, serum were collected after centrifugation at $800 \times \mathrm{g}$ and $4^{\circ} \mathrm{C}$ for $10 \mathrm{~min}$. All samples were collected under sterile conditions and stored at $-70^{\circ} \mathrm{C}$. Samples were tested in triplicate by enzyme-linked immunosorbent assay (ELISA) for assessing TGF- $\beta 1$, used commercial ELISA kits (Bioscience, San Diego, CA, USA) according to the manufacturer's instructions. The optical density at $450 \mathrm{~nm}$ was measured with an ELISA reader (Thermal scientific, Finland).

\section{RESULTS AND DISCUSSION}

A. Water contact angle of PDLLA films and PRGD/PDLLA films

TABLE 1. CONTRACT ANGLE TO WATER OF PDLLA AND

\begin{tabular}{|c|c|c|}
\multicolumn{1}{|c}{ PRGD/PDLLA FILMS $(\mathrm{N}=3, \bar{x} \pm s)$} \\
\hline & PDLLA & PRGD/PDLLA \\
\hline $\begin{array}{c}\text { Contact angle } \\
\text { to water }\left({ }^{\circ}\right)\end{array}$ & $84.5 \pm 1.5$ & $40.6 \pm 1.5$ \\
\hline
\end{tabular}

Table 1 shows the difference of contact angle to water for PDLLA and PRGD/PDLLA films. The contact angle values are calculated by averaging three measurement values in different sites of films surface. From table 1, water contact angle of the PDLLA films was larger than that of PRGD/PDLLA films. Compared with PDLLA, PRGD/PDLLA exhibited a better hydrophilicity. specimens with a layer of gold was performed using a sputter coater, and examined with SEM.

B. Proliferation of SCs on the surface of films

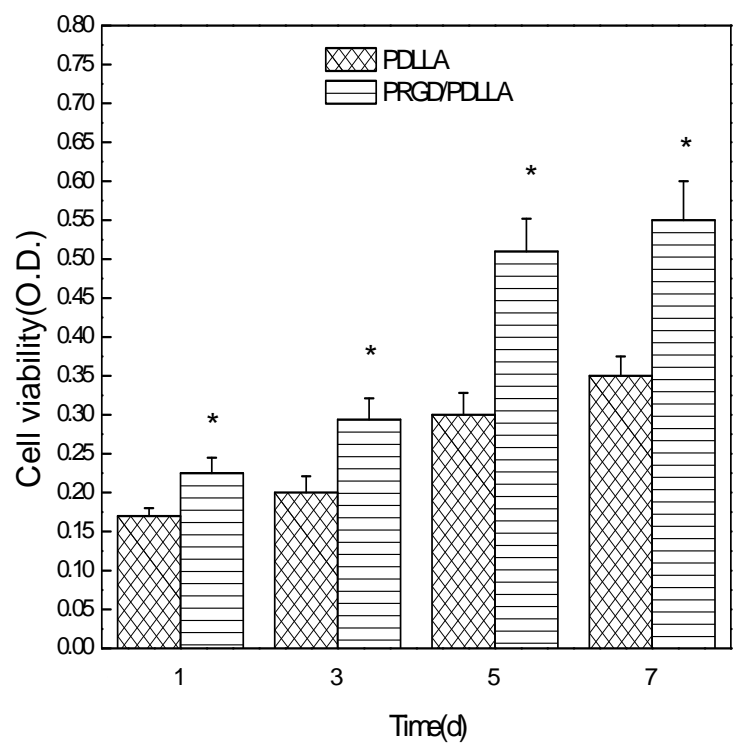

Figure 1 In vitro proliferation of SCs on films was measured by MTT assay. (*:compared with PDLLA, $\mathrm{p}<0.05$ )

MTT assay was used to examine the passage SCs proliferation on films. In MTT assay, the stable tetrazolium salts, MTT are reduced to water-soluble, orange formazan dyes by the cellular enzyme, mitochondrial dehydrogenases present in viable cells. The augmentation in enzyme activity leads to an increase in amount of formazan dye formed, which is directly proportional to the number of metabolically active cells in the culture [7].

Fig.1 showed the OD values of OECs on PRGD/PDLLA films were higher than in PDLLA culture for 2, 4 and 6 days, respectively $(\mathrm{P}<0.05)$. Thus, SCs showed PRGD/PDLLA and PDLLA films do not have any negative influence on cell growth and proliferation. Whereas, the cell proliferation was found to be significantly higher in PRGD/PDLLA films compared to PDLLA films.

C. Scanning electron microscopy of cultured SCs 

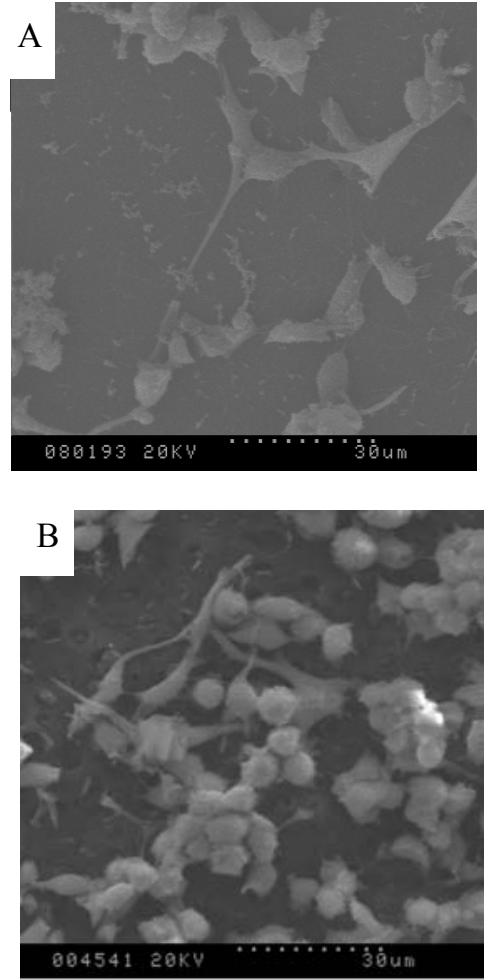

Figure 2 (A and B) Scanning electron microscope of Cultured SCs showed that cell number on PRGD/PDLLA (B) scaffolds was significantly higher than on PDLLA (A).

SCs have a quite important role in the repair of nerve system [8-10]. During the recovery of sciatic nerve injuries, SCs goes the process such as cell apoptosis, cell dedifferentiation, cell cycle arrest, and cell cycle reentry and cell differentiation [11]. The SCs morphology and cell adhesion on PRGD/PDLLA and PDLLA films were analyzed by an optical microscope (Fig.2). PRGD/PDLLA film exhibited greater cell adhesion and cell number than PDLLA film.

\section{Animal implantation and histological assessment}
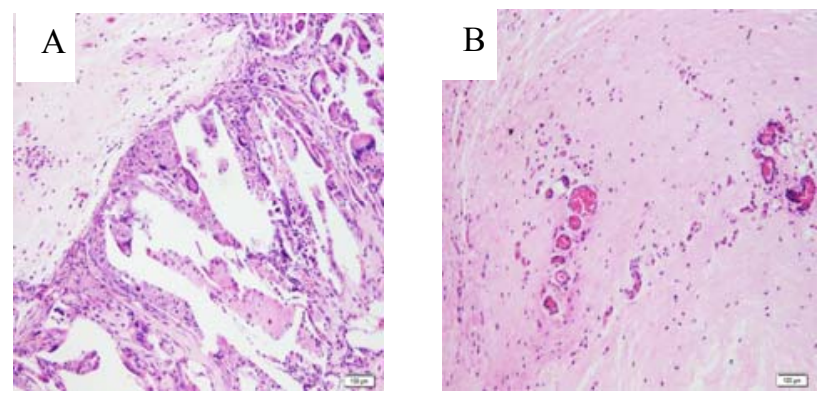

Figure.3. (A): HE staining of subcutaneous tissue after PDLLA implantation; (B): HE staining of subcutaneous tissue after PRGD/PDLLA implantation.

All experimental animals survived well without complications, including infection. The surgical wound healed normally. When the areas were re-exposed 5 weeks after surgery, PRGD/PDLLA films were found degrade as some pieces. According to HE staining, there was no inflammation phenomenon in any of the animals. Thus PRGD/PDLLA film exhibit well tissue compatibility.

\section{E. Inflammatory factors measured by ELISA}

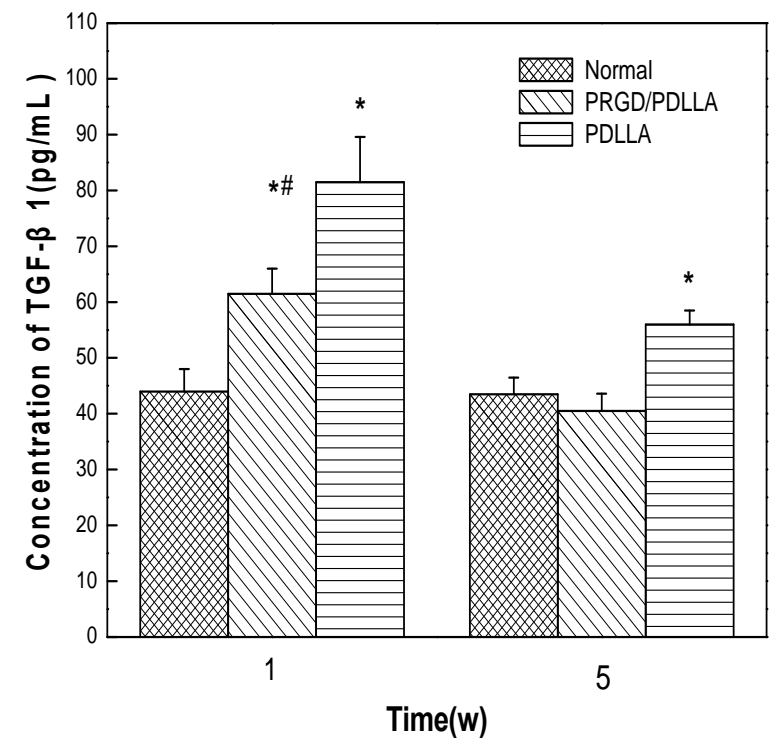

Figure. 4 The concentration of TGF- $\beta 1$ after scaffold implantation at 1,5 weeks $(\mathrm{N}=5$, \#: Compared with PRGD/PDLLA, $\mathrm{p}<0.05$;*: compared with normal, $\mathrm{p}<0.05$ )

TGF- $\beta$ also plays a major role under inflammatory conditions. TGF- $\beta$ also plays a major role under inflammatory conditions. TGF- $\beta$ in the presence of IL-6 drives the differentiation of $\mathrm{T}$ helper 17 (Th17) cells, which can promote further inflammation and augment autoimmune conditions $[12,13]$

The in vivo TGF- $\beta 1$ contents in the group of PRGD/PDLLA were lower than PDLLA group at 1week and 5 week after surgery. The TGF- $\beta 1$ contents in the PRGD/PDLLA were lower than PDLLA and has no significantly difference compared with normal rats, which suggested [14, 15] PRGD/PDLLA scaffold has lower inflammation reaction that PDLLA group in vivo.

\section{CONCLUSION}

The current study indicated that PRGD/PDLLA films can promote proliferation of SCs. PRGD/PDLLA had better biocompatibility for SCs compared with PDLLA films. The result of animal implantation and histological assessment showed that PRGD/PDLLA film has good tissue compatibility and degradation property. Therefore, PRGD/PDLLA films could be used as one kinds of nerve repairing scaffolds. The present study may provide a new approach for nerve injury treatment.

\section{ACKNOWLEDGMENT}

This work was supported by the National Basic Research program (2011CB606205) and National Natural Science Foundation of China (No. 51403168) ; Natural 
Science Foundation of Hubei Province (No. 2013CFB354) and Central University grants (No.2013-IV-099).

\section{REFERENCES}

[1] V. Maquet, D. Martin, F. Scholtes, R.Franzen, "Poly(D,L-lactide) foams modified by poly(ethylene oxide)-block-poly(D,L-lactide) copolymers and a-FGF: in vitro and in vivo evaluation for spinal cord regeneration," Biomaterials, vol.22, ,2001, pp. 1137-1146.

[2] Gu X, Ding F, Yang Y,"Construction of tissue engineered nerve grafts and their application in peripheral nerve regeneration." Prog Neurobiol, 2011, 93:204-230.

[3] F.W. David, "The role of short synthetic adhesion peptides in regenerative medicine; The debate", Biomaterials, vol, 32, 2011,pp 4195-4197,.

[4] Q.J. Yan, J. Li, S.P. Li, "Synthesis and RGD Peptide Modification of Poly\{(lactic acid)-co-[(glycolic acid )-alt-(L-lysine)]\}," ePolymers, 028, 2008, pp. 1-12.

[5] Yu-Sheng Hsueh, S. Savitha, S. Sadhasivam,'Design and synthesis of elastin-like polypeptides for an ideal nerve conduit in peripheral nerve regeneration." Materials Science and Engineering, 2014, (38):119-126.

[6] YH Wang, YX Yin, HL Dai, SP Li, "Evaluation of a Novel Bioabsorbable PRGD/PDLLA/ $/$-TCP/NGF Composites in Repair of Peripheral Nerves", Journal of Wuhan University of Technolotgy-Mater. Sci. Ed. , vol, 24, 2009,pp. 409-414.

[7] D.P. Barry, B.L. Beaman, "Modulation of eukaryotic cell apoptosis by members of the bacterial order Actinomycetales. "Apoptosis, vol.11,2006,pp.1695-1707.
[8] Fouad K, Schnell L, Bunge M B, "Combining Schwann cell bridges and olfactory-ensheathing glia grafts with chondroitinase promotes locomotor recovery after complete transection of the spinal cord.'The Journal of Neuroscience,vol. 25, 2005,pp.11691178 .

[9] Mosahebi A, Fuller P, Wiberg M, "Effect of allogeneic Schwann cell transplantation on peripheral nerve regeneration."Experimental neurology,vol. 173, 2002, pp. 213-223.

[10] Rodríguez F J, Verdú E, Ceballos D, "Nerve guides seeded with autologous Schwann cells improve nerve regeneration."Experimental neurology,vol. 161, 2000,pp. 571-584.

[11] J.C. Liu, S.C. Heilshorn, D.A. Tirrell. "Comparative cell response to artificial extracellular matrix proteins containing the RGD and CS5 cell-binding domains. "Biomacromolecules, 2004, pp.497-504.

[12] H.S Taskinen, T Olsson, A Bucht, " Peripheral nerve injury induces endoneurial expression of IFN-g, IL-10and TNF-a mRNA.”Journal of Neuroimmunology,vol. 102, 2000,pp. 17-25.

[13] Jones K S. Effects of biomaterial-induced inflammation on fibrosis and rejection[C]//Seminars in immunology. Academic Press, 2008, 20(2): pp. 130-136.

[14] Thevenot P T, Nair A M, Shen J, et al. The effect of incorporation of SDF-1 $\alpha$ into PLGA scaffolds on stem cell recruitment and the inflammatory response[J]. Biomaterials, vol. 31,2010, pp. 39974008

[15] Geetha M, Singh A K, Asokamani R, et al. Ti based biomaterials, the ultimate choice for orthopaedic implants-a review [J]. Progress in Materials Science, vol.54,2009,pp. 397-425. 